\title{
Contributions to the Knowledge on the Amphibians and Reptiles of Ialomița County (South-Eastern Romania)
}

\author{
Alexandru IFTIME ${ }^{1, *}$, Oana IFTIME ${ }^{2}$ \\ 1“"Grigore Antipa” National Museum of Natural History, 1 Kiseleff Blvd, 011341 Bucharest, Romania. \\ ${ }^{2}$ Department of Genetics, Faculty of Biology, University of Bucharest, Aleea Portocalelor 1-3, 060101 \\ Bucharest 6, Romania. \\ *corresponding author, e-mail: aiftime@antipa.ro
}

Received: September 22, 2017; Accepted: November 20, 2017; Available online: December 29, 2017; Printed: December 31, 2017

\begin{abstract}
The results of faunistical and ecological surveys of the amphibians in Ialomita County (Romania) are presented here; 12 amphibian species were identified, two amphibian hybrids and five reptile species, with new records for many of those.
\end{abstract}

Key words: Ialomița County, amphibians, reptiles, localities, distribution.

\section{INTRODUCTION}

Although the last decades have seen a general flourishing of herpetological studies in Romania, Ialomita County is among the least investigated area in terms of distribution records for amphibian and reptile species. The analysis of existant records (Fuhn, 1960; Fuhn \& Vancea, 1961; Iftime \& Iftime, 2007; Covaciu-Marcov et al., 2009; Sos, 2011; Cogălniceanu et al., 2013a, b; Székely et al., 2013; Sahlean et al., 2014; Török, 2014; Wielstra et al., 2016), shows that although despite the records of numerous species (Triturus dobrogicus, Lissotriton vulgaris, Bombina bombina, Pelobates fuscus, P. syriacus, Bufo bufo, Bufotes viridis, Hyla orientalis, Pelophylax ridibundus/P. kl. esculentus, P. lessonae, Emys orbicularis, Lacerta agilis, L. viridis, Podarcis tauricus, Dolichophis caspius, Natrix natrix, N. tessellata), practically all of them are concentrated in the Danube floodplain and its immediate vicinity in the east of the county (Török, 2001; Covaciu-Marcov et al., 2009; Cogălniceanu et al., 2013a, b); the few data pertaining to the western and central Ialomitia plain are generally old, with the exception of a string of new records of Emys orbicularis along Ialomița River (Sos, 2011; Cogălniceanu et al., 2013b). We present new records of herpetofaunal species from Ialomita County (in order to concentrate on the less investigated Ialomita valley plain, we excluded "Balta Ialomiței", an area of largely modified floodplain enclosed between two arms of the Danube).

\section{MATERIALS AND METHODS}

This paper is based upon field work performed in June 2011, August 2015, March-July 2016 and March 2017 (the search effort not being equal in all investigated points, nor necessarily adequate for the detectability of all potentially present species, absence of a species from a point should not be considered as absolute). The recordings summarizing 105 points that can be grouped into 76 localities or study areas (see table 1; there may be more than one recording point per locality). Qualitative observations 
were undertaken using the active transects method, combining visual and acoustic identification (after Heyer et al., 1994, and McDiarmid, 1992, in Cogălniceanu, 1997), the transect being $4 \mathrm{~m}$ wide. For the genus Rana, subgenus Pelophylax (i.e. "Green Frogs" or "West Palaearctic Water Frogs") specific identification was performed using morphological and morphometrical characteristics and song-based identification if possible. Photographs were taken whenever possible.

\section{RESULTS}

location.

The results are included in table 1, with species found tabulated for every

Locations and species (note: GPS altitude indications below $10 \mathrm{~m}$ were

Table 1 noted as such and not given exactly, they are likely imprecise since the

lowest altitudes in the county are ca. 7-10 m - Mâciu et al., 1982).

\begin{tabular}{|c|c|c|c|}
\hline Locality & Coordinates & & Observed species/hybrids \\
\hline $\begin{array}{l}\text { A2 highway near } \\
\text { Fetești }\end{array}$ & $\begin{array}{l}\text { N44 } 23.867 \\
\text { E27 } 48.963\end{array}$ & $47 \mathrm{~m}$ & Dolichophis caspius \\
\hline Coșereni & $\begin{array}{l}\text { N } 4440.578 \\
\text { E } 2633.306\end{array}$ & $45 \mathrm{~m}$ & Dolichophis caspius \\
\hline Coșereni & $\begin{array}{l}\text { N44 } 41.904 \\
\text { E26 } 34.313\end{array}$ & $45 \mathrm{~m}$ & Rana dalmatina, Pelophylax ridibundus \\
\hline Piersica & $\begin{array}{l}\text { N44 } 33.564 \\
\text { E27 } 00.538\end{array}$ & $49 \mathrm{~m}$ & $\begin{array}{l}\text { Triturus dobrogicus, Lissotriton vulgaris, Bombina } \\
\text { bombina, Pelophylax ridibundus, P. k1. esculentus, Lacerta } \\
\text { agilis, L. viridis, Dolichophis caspius }\end{array}$ \\
\hline Piersica & $\begin{array}{l}\text { N44 } 34.071 \\
\text { E26 } 59.858\end{array}$ & $34 \mathrm{~m}$ & Bombina bombina, Bufotes viridis, Pelophylax ridibundus \\
\hline Piersica & $\begin{array}{l}\text { N44 } 33.930 \\
\text { E27 } 00.648\end{array}$ & $30 \mathrm{~m}$ & Lissotriton vulgaris, Bombina bombina, Hyla orientalis \\
\hline Axintele & $\begin{array}{l}\text { N44 } 37.077 \\
\text { E26 } 43.063\end{array}$ & $66 \mathrm{~m}$ & $\begin{array}{l}\text { Bombina bombina, Pelobates fuscus, P. syriacus, Bufotes } \\
\text { viridis, Pelophylax ridibundus, Lacerta viridis }\end{array}$ \\
\hline Axintele & $\begin{array}{l}\text { N44 } 36.887 \\
\text { E26 } 44.241\end{array}$ & $47 \mathrm{~m}$ & $\begin{array}{l}\text { Bombina bombina, Pelobates fuscus, Bufotes viridis, } \\
\text { Pelophylax ridibundus }\end{array}$ \\
\hline Axintele & $\begin{array}{l}\text { N } 4436.279 \\
\text { E } 2645.638\end{array}$ & ca. $50 \mathrm{~m}$ & Pelophylax ridibundus, Lacerta viridis, L. agilis \\
\hline $\begin{array}{l}\text { Crăsanii de Sus - } \\
\text { Călugărului forest }\end{array}$ & $\begin{array}{l}\text { N44 } 35.893 \\
\text { E26 } 48.856\end{array}$ & $75 \mathrm{~m}$ & Lacerta viridis \\
\hline Sărățeni & $\begin{array}{l}\text { N44 } 37.793 \\
\text { E26 } 54.232\end{array}$ & $36 \mathrm{~m}$ & $\begin{array}{l}\text { Triturus dobrogicus, Lissotriton vulgaris, Bombina } \\
\text { bombina, Pelophylax ridibundus }\end{array}$ \\
\hline Sărățeni & $\begin{array}{l}\text { N } 4437.537 \\
\text { E } 2654.899\end{array}$ & $36 \mathrm{~m}$ & $\begin{array}{l}\text { Triturus dobrogicus, Bombina bombina, Pelobates fuscus, } \\
\text { Dolichophis caspius }\end{array}$ \\
\hline Sărățeni & $\begin{array}{l}\text { N } 4437.500 \\
\text { E } 2656.305\end{array}$ & $36 \mathrm{~m}$ & $\begin{array}{l}\text { Bombina bombina, Pelophylax ridibundus, Lacerta agilis, } \\
\text { Natrix natrix }\end{array}$ \\
\hline Căzănești & $\begin{array}{l}\text { N44 } 37.661 \\
\text { E26 } 58.608\end{array}$ & $32 \mathrm{~m}$ & $\begin{array}{l}\text { Triturus dobrogicus, Lissotriton vulgaris, Bombina } \\
\text { bombina, Pelobates fuscus, P. syriacus, Hyla orientalis, } \\
\text { Pelophylax ridibundus }\end{array}$ \\
\hline Orezu & $\begin{array}{l}\text { N44 } 35.441 \\
\text { E27 } 02.363\end{array}$ & $29 \mathrm{~m}$ & $\begin{array}{l}\text { Triturus dobrogicus, Lissotriton vulgaris, Bombina } \\
\text { bombina, Hyla orientalis, Rana dalmatina, Pelophylax } \\
\text { ridibundus, Lacerta viridis, Dolichophis caspius }\end{array}$ \\
\hline Marsilieni & $\begin{array}{l}\text { N44 } 32.592 \\
\text { E27 } 04.887\end{array}$ & $27 \mathrm{~m}$ & Bombina bombina, Hyla orientalis, Pelophylax ridibundus \\
\hline
\end{tabular}


Table 1 (continued)

\begin{tabular}{|c|c|c|}
\hline Locality & Coordinates & Observed species/hybrids \\
\hline Marsilieni & $\begin{array}{l}\text { N44 } 32.169 \\
\text { E27 } 04.793\end{array}$ & Bombina bombina, Pelobates fuscus, Pelophylax ridibundus \\
\hline Ciochina & $\begin{array}{l}\text { N44 } 34.926 \\
\text { E27 } 02.918\end{array}$ & $\begin{array}{l}\text { Triturus dobrogicus, Lissotriton vulgaris, Bombina } \\
\text { bombina, Hyla orientalis, Rana dalmatina, Pelophylax } \\
\text { ridibundus, P. kl. esculentus ?, Lacerta viridis, Dolichophis } \\
\text { caspius }\end{array}$ \\
\hline Borduşelu & $\begin{array}{l}\text { N44 } 32.724 \\
\text { E27 } 04.001\end{array}$ & $\begin{array}{l}\text { Bombina bombina, Pelobates fuscus, Bufotes viridis, } \\
\text { Pelophylax ridibundus, Dolichophis caspius }\end{array}$ \\
\hline Albești/Buești & $\begin{array}{l}\text { N44 } 32.559 \\
\text { E27 } 09.738\end{array}$ & $\begin{array}{l}\text { Triturus dobrogicus, Bombina bombina, Bufotes viridis, } \\
\text { Hyla orientalis, Pelophylax ridibundus, P. lessonae, Lacerta } \\
\text { viridis, Natrix natrix }\end{array}$ \\
\hline Bucu/Mărculești & $\begin{array}{l}\text { N44 } 34.950 \\
\text { E27 } 30.370\end{array}$ & Lacerta viridis \\
\hline Scânteia & $\begin{array}{l}\text { N44 } 42.129 \\
\text { E27 } 28.553\end{array}$ & Bombina bombina, Bufotes viridis \\
\hline Scânteia & $\begin{array}{l}\text { N44 } 42.899 \\
\text { E27 } 28.237\end{array}$ & Pelophylax ridibundus \\
\hline Iazu & $\begin{array}{l}\text { N44 } 43.842 \\
\text { E27 } 24.144\end{array}$ & Bombina bombina, Bufotes viridis, Pelophylax ridibundus \\
\hline Iazu & $\begin{array}{l}\text { N44 } 42.209 \\
\text { E27 } 25.141\end{array}$ & Natrix natrix \\
\hline Smirna & $\begin{array}{l}\text { N44 } 43.177 \\
\text { E27 } 20.331\end{array}$ & Bufotes viridis \\
\hline Malu & $\begin{array}{l}\text { N } 4439.054 \\
\text { E } 2649.132\end{array}$ & Bufotes viridis \\
\hline Ion Roată & $\begin{array}{l}\text { N } 4439.774 \\
\text { E } 2647.372\end{array}$ & Bufotes viridis \\
\hline Broșteni & $\begin{array}{l}\text { N44 } 40.139 \\
\text { E26 } 45.076\end{array}$ & Bufotes viridis, Pelophylax ridibundus \\
\hline Stelnica & $\begin{array}{l}\text { N44 } 24.812 \\
\text { E27 } 53.255 \text { bellow } 10 \mathrm{~m}\end{array}$ & $\begin{array}{l}\text { Bombina bombina, Pelobates fuscus, Bufotes viridis, } \\
\text { Pelophylax ridibundus, Lacerta viridis }\end{array}$ \\
\hline Stelnica & $\begin{array}{l}\text { N44 } 25.559 \\
\text { E27 55.260 bellow } 10 \mathrm{~m}\end{array}$ & $\begin{array}{l}\text { Bombina bombina, Bufotes viridis, Pelophylax ridibundus, } \\
\text { P. kl. esculentus }\end{array}$ \\
\hline Stelnica & $\begin{array}{l}\text { N44 } 25.912 \\
\text { E27 } 55.382 \text { bellow } 10 \mathrm{~m}\end{array}$ & $\begin{array}{l}\text { Bombina bombina, Pelobates fuscus, Bufotes viridis, } \\
\text { Pelophylax ridibundus, } P \text {. kl. esculentus }\end{array}$ \\
\hline Cegani & $\begin{array}{l}\text { N44 } 26.472 \\
\text { E27 } 55.433 \text { bellow } 10 \mathrm{~m}\end{array}$ & $\begin{array}{l}\text { Bombina bombina, Pelophylax ridibundus, } P . \text { k1. esculentus, } \\
\text { P. lessonae }\end{array}$ \\
\hline Cegani & $\begin{array}{l}\text { N44 } 26.683 \\
\text { E27 } 55.645 \text { bellow } 10 \mathrm{~m}\end{array}$ & Bombina bombina, Bufotes viridis, Pelophylax ridibundus \\
\hline Cegani & $\begin{array}{l}\text { N44 } 26.802 \\
\text { E27 55.275 bellow } 10 \mathrm{~m}\end{array}$ & $\begin{array}{l}\text { Lissotriton vulgaris, Bombina bombina, Pelophylax } \\
\text { ridibundus, Natrix natrix }\end{array}$ \\
\hline Cegani & $\begin{array}{l}\text { N44 } 27.233 \\
\text { E27 } 54.477 \text { bellow } 10 \mathrm{~m}\end{array}$ & $\begin{array}{l}\text { Triturus dobrogicus, Lissotriton vulgaris, Bombina } \\
\text { bombina, Bufotes viridis }\end{array}$ \\
\hline Borduşani & $\begin{array}{l}\text { N44 } 28.635 \\
\text { E27 } 54.625 \text { bellow } 10 \mathrm{~m}\end{array}$ & $\begin{array}{l}\text { Triturus dobrogicus, Lissotriton vulgaris, Bombina bombina, } \\
\text { Bufotes viridis, Hyla arborea, Pelophylax ridibundus }\end{array}$ \\
\hline Borduşani & $\begin{array}{l}\text { N44 } 28.544 \\
\text { E27 } 55.369 \text { bellow } 10 \mathrm{~m}\end{array}$ & $\begin{array}{l}\text { Triturus dobrogicus, Lissotriton vulgaris, Bombina } \\
\text { bombina, Bufotes viridis, Natrix natrix }\end{array}$ \\
\hline
\end{tabular}


Table 1 (continued)

\begin{tabular}{|c|c|c|}
\hline Locality & Coordinates & Observed species/hybrids \\
\hline Borduşani & $\begin{array}{l}\text { N44 } 28.431 \\
\text { E27 } 55.842 \text { bellow } 10 \mathrm{~m}\end{array}$ & Triturus dobrogicus, Lissotriton vulgaris, Bombina bombina \\
\hline Progresu & $\begin{array}{l}\text { N44 } 35.079 \\
\text { E27 } 52.857 \text { bellow } 10 \mathrm{~m}\end{array}$ & Bombina bombina, Bufotes viridis, Pelophylax ridibundus \\
\hline Progresu & $\begin{array}{l}\text { N44 35.369 } \\
\text { E27 } 53.144 \text { bellow } 10 \mathrm{~m}\end{array}$ & $\begin{array}{l}\text { Lissotriton vulgaris, Bombina bombina, Pelophylax } \\
\text { ridibundus, Emys orbicularis, Lacerta viridis, L. agilis }\end{array}$ \\
\hline Progresu & $\begin{array}{l}\text { N44 } 35.672 \\
\text { E27 } 53.412 \text { bellow } 10 \mathrm{~m}\end{array}$ & Pelophylax ridibundus, Lacerta viridis, L. agilis \\
\hline Progresu & $\begin{array}{l}\text { N44 } 36.367 \\
\text { E27 } 54.106 \text { bellow } 10 \mathrm{~m}\end{array}$ & $\begin{array}{l}\text { Lissotriton vulgaris, Bombina bombina, Bufotes viridis, } \\
\text { Pelophylax ridibundus, Podarcis taurica, Lacerta viridis }\end{array}$ \\
\hline Movila & $\begin{array}{l}\text { N44 } 30.938 \\
\text { E27 } 44.119\end{array}$ & Lacerta viridis, Natrix natrix \\
\hline Movila & $\begin{array}{l}\text { N44 } 32.263 \\
\text { E27 } 40.959\end{array}$ & Lacerta viridis \\
\hline $\begin{array}{l}\text { Mihail } \\
\text { Kogălniceanu }\end{array}$ & $\begin{array}{l}\text { N44 } 39.993 \\
\text { E27 } 42.187 \text { bellow } 10 \mathrm{~m}\end{array}$ & Bufotes viridis, Hyla orientalis, Pelophylax ridibundus \\
\hline Gura Ialomiței & $\begin{array}{l}\text { N44 } 42.116 \\
\text { E27 } 45.769 \text { bellow } 10 \mathrm{~m}\end{array}$ & $\begin{array}{l}\text { Bombina bombina, Bufotes viridis, Pelobates syriacus, } \\
\text { Pelophylax ridibundus, } P . \mathrm{kl} \text {. esculentus, Lacerta agilis, } \\
\text { Natrix natrix }\end{array}$ \\
\hline Luciu & $\begin{array}{l}\text { N44 } 45.375 \\
\text { E27 43.471 bellow } 10 \mathrm{~m}\end{array}$ & $\begin{array}{l}\text { Bombina bombina, Bufotes viridis, Pelobates fuscus, } P \text {. } \\
\text { syriacus }\end{array}$ \\
\hline $\begin{array}{l}\text { Oraşu de Floci } \\
\text { Archeological } \\
\text { Reservation }\end{array}$ & $\begin{array}{l}\text { N44 } 41.586 \\
\text { E27 } 49.320 \text { bellow } 10 \mathrm{~m}\end{array}$ & $\begin{array}{l}\text { Bombina bombina, Pelophylax ridibundus, P. kl. esculentus, } \\
\text { Emys orbicularis }\end{array}$ \\
\hline Giurgeni & $\begin{array}{l}\text { N44 } 42.177 \\
\text { E27 } 51.129 \text { bellow } 10 \mathrm{~m}\end{array}$ & $\begin{array}{l}\text { Lissotriton vulgaris, Bombina bombina, Bufotes viridis, } \\
\text { Hyla orientalis, Pelophylax ridibundus, P. lessonae, Lacerta } \\
\text { agilis }\end{array}$ \\
\hline Țăndărei & $\begin{array}{l}\text { N44 38.334 } \\
\text { E27 37.451 bellow } 10 \mathrm{~m}\end{array}$ & Bombina bombina \\
\hline Ograda & $\begin{array}{l}\text { N44 } 37.357 \\
\text { E27 } 35.065 \text { bellow } 10 \mathrm{~m}\end{array}$ & $\begin{array}{l}\text { Triturus dobrogicus, Lissotriton vulgaris, Bombina } \\
\text { bombina, Pelobates fuscus, Pelophylax ridibundus, } P . \mathrm{kl} . \\
\text { esculentus }\end{array}$ \\
\hline Slobozia & $\begin{array}{l}\text { N44 } 34.915 \\
\text { E27 } 20.194 \\
\end{array}$ & $\begin{array}{l}\text { Bombina bombina, Pelophylax ridibundus, Emys } \\
\text { orbicularis, Lacerta agilis }\end{array}$ \\
\hline Amara & $\begin{array}{l}\text { N44 } 36.324 \\
\text { E27 } 19.763\end{array}$ & Lacerta viridis, Lacerta agilis \\
\hline Grivița & \begin{tabular}{|l|} 
N44 43.325 \\
E27 18.286 \\
\end{tabular} & Pelophylax ridibundus \\
\hline Miloșești & $\begin{array}{l}\text { N44 } 41.873 \\
\text { E27 } 12.588\end{array}$ & $\begin{array}{l}\text { Triturus dobrogicus, Lissotriton vulgaris, Bombina } \\
\text { bombina, Pelophylax ridibundus }\end{array}$ \\
\hline Gheorghe Doja & \begin{tabular}{|l|l|} 
N44 36.883 \\
E27 09.724 \\
\end{tabular} & Bufotes viridis, Emys orbicularis \\
\hline Cocora & $\begin{array}{l}\text { N44 } 43.456 \\
\text { E27 } 03.295 \\
\end{array}$ & $\begin{array}{l}\text { Bombina bombina, Bufotes viridis, Pelophylax ridibundus, } \\
\text { P. kl. esculentus }\end{array}$ \\
\hline Cocora & $\begin{array}{l}\text { N44 } 44.865 \\
\text { E27 } 01.725\end{array}$ & $\begin{array}{l}\text { Triturus dobrogicus, Lissotriton vulgaris, Bombina } \\
\text { bombina, Bufotes viridis, Lacerta agilis }\end{array}$ \\
\hline Colelia & $\begin{array}{l}\text { N44 } 45.433 \\
\text { E26 } 59.005\end{array}$ & Bombina bombina, Pelophylax ridibundus \\
\hline
\end{tabular}


Table 1 (continued)

\begin{tabular}{|c|c|c|c|}
\hline Locality & Coordinates & & Observed species/hybrids \\
\hline Valea Măcrişului & $\begin{array}{l}\text { N44 } 44.310 \\
\text { E26 } 50.593\end{array}$ & $54 \mathrm{~m}$ & $\begin{array}{l}\text { Triturus dobrogicus, Bombina bombina, Bufotes viridis, } \\
\text { Pelobates fuscus }\end{array}$ \\
\hline Movilița & $\begin{array}{l}\text { N44 } 37.953 \\
\text { E26 } 27.573\end{array}$ & $68 \mathrm{~m}$ & $\begin{array}{l}\text { Bufotes viridis, Pelophylax ridibundus, P. kl. esculentus } \\
\text { Lacerta viridis }\end{array}$ \\
\hline $\begin{array}{l}\text { Movilița - } \\
\text { Stroiasca I forest }\end{array}$ & $\begin{array}{l}\text { N44 } 39.009 \\
\text { E26 } 26.753\end{array}$ & $72 \mathrm{~m}$ & Bufotes viridis?, Rana dalmatina \\
\hline $\begin{array}{l}\text { Movilița - } \\
\text { Stroiasca II forest }\end{array}$ & $\begin{array}{l}\text { N } 4438.469 \\
\text { E } 2625.997\end{array}$ & ca. $70 \mathrm{~m}$ & Bufotes viridis, Lacerta viridis \\
\hline $\begin{array}{l}\text { Dridu - Stroiasca } \\
\text { I forest }\end{array}$ & $\begin{array}{l}\text { N44 } 40.128 \\
\text { E26 } 26.393\end{array}$ & $70 \mathrm{~m}$ & Bufotes viridis, Rana dalmatina \\
\hline Dridu & $\begin{array}{l}\text { N44 } 42.403 \\
\text { E26 } 28.464\end{array}$ & $61 \mathrm{~m}$ & $\begin{array}{l}\text { Bufotes viridis, Pelophylax ridibundus, P. kl. esculentus } \\
\text { Lacerta agilis }\end{array}$ \\
\hline Fierbinți-Târg & $\begin{array}{l}\text { N44 40.716 } \\
\text { E26 } 24.379\end{array}$ & $62 \mathrm{~m}$ & $\begin{array}{l}\text { Bombina bombina, Bufotes viridis, Pelophylax ridibundus, } \\
\text { Natrix natrix }\end{array}$ \\
\hline Rădulești (Brazii) & $\begin{array}{l}\text { N44 } 45.885 \\
\text { E26 } 21.784\end{array}$ & $72 \mathrm{~m}$ & Pelophylax ridibundus, $P$. lessonae, $P$. kl. esculentus \\
\hline $\begin{array}{l}\text { Near Rădulești } \\
\text { (Brazii) - Prahova } \\
\text { county, on limit }\end{array}$ & $\begin{array}{l}\text { N44 } 46.232 \\
\text { E26 } 22.325\end{array}$ & $69 \mathrm{~m}$ & Bombina variegata \\
\hline Rădulești (Brazii) & $\begin{array}{l}\text { N44 } 45.702 \\
\text { E26 } 22.136\end{array}$ & $65 \mathrm{~m}$ & $\begin{array}{l}\text { Bombina bombina, Pelophylax ridibundus, } P \text {. lessonae, } P \text {. } \\
\text { kl. esculentus, Emys orbicularis, Lacerta viridis }\end{array}$ \\
\hline Patru Frați & $\begin{array}{l}\text { N44 } 44.629 \\
\text { E26 } 27.764\end{array}$ & $56 \mathrm{~m}$ & Lacerta viridis \\
\hline $\begin{array}{l}\text { Moldoveni - } \\
\text { Leaşa Forest }\end{array}$ & $\begin{array}{l}\text { N44 } 42.998 \\
\text { E26 } 29.345\end{array}$ & $53 \mathrm{~m}$ & Pelophylax ridibundus, Lacerta viridis \\
\hline $\begin{array}{l}\text { Moldoveni- } \\
\text { Coșereni }\end{array}$ & $\begin{array}{l}\text { N } 4442.720 \\
\text { E } 2632.581\end{array}$ & ca. $50 \mathrm{~m}$ & Lacerta viridis \\
\hline $\begin{array}{l}\text { Moldoveni- } \\
\text { Coșereni }\end{array}$ & $\begin{array}{l}\text { N } 4442.811 \\
\text { E } 2632.108\end{array}$ & ca. $50 \mathrm{~m}$ & Rana dalmatina, Pelophylax ridibundus, Triturus cristatus \\
\hline Bărbulești & $\begin{array}{l}\text { N44 } 43.284 \\
\text { E26 } 36.259\end{array}$ & $39 \mathrm{~m}$ & Pelophylax ridibundus, Lacerta agilis \\
\hline Armășești & $\begin{array}{l}\text { N44 } 44.483 \\
\text { E26 } 34.463\end{array}$ & $42 \mathrm{~m}$ & $\begin{array}{l}\text { Triturus cristatus } \mathrm{x} \text { T. dobrogicus, Lissotriton vulgaris, } \\
\text { Bombina bombina, }\end{array}$ \\
\hline Slătioarele & $\begin{array}{l}\text { N44 } 46.981 \\
\text { E26 } 31.401\end{array}$ & $51 \mathrm{~m}$ & Pelophylax ridibundus \\
\hline Adâncata & $\begin{array}{l}\text { N44 } 46.765 \\
\text { E26 } 26.884\end{array}$ & $25 \mathrm{~m}$ & Bombina bombina, Bufotes viridis, Lacerta viridis \\
\hline Rodeanu Lake & $\begin{array}{l}\text { N44 } 47.034 \\
\text { E26 } 29.477\end{array}$ & $53 \mathrm{~m}$ & Bombina bombina, Bufotes viridis \\
\hline Roșiori & $\begin{array}{l}\text { N44 } 36.378 \\
\text { E26 } 32.637\end{array}$ & $56 \mathrm{~m}$ & $\begin{array}{l}\text { Bombina bombina, Pelobates fuscus, Bufotes viridis, } \\
\text { Pelophylax ridibundus, } P \text {. kl. esculentus, Emys orbicularis }\end{array}$ \\
\hline Drăgoești & $\begin{array}{l}\text { N44 } 34.290 \\
\text { E26 } 32.640\end{array}$ & $44 \mathrm{~m}$ & Bufotes viridis, Pelophylax ridibundus \\
\hline Chiroiu & $\begin{array}{l}\text { N44 } 35.966 \\
\text { E26 } 30.736\end{array}$ & $53 \mathrm{~m}$ & Pelophylax ridibundus \\
\hline Livedea & $\begin{array}{l}\text { N44 } 35.404 \\
\text { E26 } 25.539\end{array}$ & $59 \mathrm{~m}$ & Bombina bombina, Pelophylax ridibundus \\
\hline
\end{tabular}


Table 1 (continued)

\begin{tabular}{|c|c|c|}
\hline Locality & Coordinates & Observed species/hybrids \\
\hline Livedea & $\begin{array}{l}\text { N44 } 35.201 \\
\text { E26 25.741 }\end{array}$ & Bombina bombina, Pelophylax ridibundus, Emys orbicularis \\
\hline Hagiești & $\begin{array}{l}\text { N44 } 32.891 \\
\text { E26 } 25.718\end{array}$ & Pelophylax ridibundus, Emys orbicularis, Natrix natrix \\
\hline Boteni & $\begin{array}{l}\text { N44 } 33.976 \\
\text { E26 } 26.861\end{array}$ & Pelophylax ridibundus \\
\hline Lilieci & $\begin{array}{l}\text { N44 } 32.388 \\
\text { E26 } 23.338\end{array}$ & Lacerta agilis \\
\hline Lilieci/Cătrunești & $\begin{array}{l}\text { N44 } 33.437 \\
\text { E26 } 22.907\end{array}$ & Pelophylax ridibundus, Emys orbicularis \\
\hline $\begin{array}{l}\text { Urziceni-Cotorca } \\
\text { Lake }\end{array}$ & $\begin{array}{l}\text { N44 } 44.228 \\
\text { E26 } 38.709\end{array}$ & Pelophylax ridibundus, Lacerta viridis \\
\hline $\begin{array}{l}\text { Munteni-Buzău - } \\
\text { Sărățuica Lake }\end{array}$ & $\begin{array}{l}\text { N44 } 38.476 \\
\text { E26 } 57.062\end{array}$ & Bombina bombina, Pelophylax ridibundus, Natrix natrix \\
\hline $\begin{array}{l}\text { Munteni-Buzău - } \\
\text { wetland Sărățuica } \\
\text { Mică }\end{array}$ & $\begin{array}{l}\text { N44 } 37.763 \\
\text { E26 } 57.017\end{array}$ & $\begin{array}{l}\text { Bombina bombina, Bufotes viridis, Pelobates fuscus, } P \text {. } \\
\text { syriacus, Hyla orientalis, Pelophylax ridibundus }\end{array}$ \\
\hline Fundata & $\begin{array}{l}\text { N44 } 34.878 \\
\text { E27 } 15.620\end{array}$ & Bombina bombina, Pelophylax ridibundus, Lacerta agilis \\
\hline $\begin{array}{l}\text { Valea Ciorii - } \\
\text { Strachina Lake }\end{array}$ & $\begin{array}{l}\text { N44 } 42.860 \\
\text { E27 } 32.085 \text { bellow } 10 \mathrm{~m}\end{array}$ & $\begin{array}{l}\text { Bombina bombina, Bufotes viridis, Pelobates fuscus, } \\
\text { Pelophylax ridibundus }\end{array}$ \\
\hline Platonești & $\begin{array}{l}\text { N44 } 35.897 \\
\text { E27 } 41.541\end{array}$ & Bufotes viridis, Lacerta viridis, L. agilis \\
\hline Hagieni & $\begin{array}{l}\text { N44 } 39.753 \\
\text { E27 } 45.069 \text { bellow } 10 \mathrm{~m}\end{array}$ & Pelophylax ridibundus \\
\hline Hagieni-Vlădeni & $\begin{array}{l}\text { N44 } 38.687 \\
\text { E27 } 49.238 \text { bellow } 10 \mathrm{~m}\end{array}$ & Bufotes viridis, Natrix natrix f. bilineata \\
\hline Vlădeni & $\begin{array}{l}\text { N44 } 38.040 \\
\text { E27 } 49.607 \text { bellow } 10 \mathrm{~m}\end{array}$ & $\begin{array}{l}\text { Lissotriton vulgaris, Bombina bombina, Pelophylax } \\
\text { ridibundus, Lacerta viridis }\end{array}$ \\
\hline Vlădeni & $\begin{array}{l}\text { N } 4436.672 \\
\text { E } 2752.174 \text { bellow } 10 \mathrm{~m}\end{array}$ & Pelophylax ridibundus, Lacerta agilis, Dolichophis caspius \\
\hline Vlădeni & $\begin{array}{l}\text { N } 4436.156 \\
\text { E } 2751.762 \text { bellow } 10 \mathrm{~m}\end{array}$ & Lacerta agilis \\
\hline Țăndărei-Săveni & $\begin{array}{l}\text { N44 37.272 } \\
\text { E27 39.242 bellow } 10 \mathrm{~m}\end{array}$ & $\begin{array}{l}\text { Triturus dobrogicus, Bombina bombina, Bufotes viridis, } \\
\text { Pelobates fuscus, Pelophylax ridibundus, P. kl. esculentus }\end{array}$ \\
\hline Sudiți & $\begin{array}{l}\text { N44 } 35.086 \\
\text { E27 36.244 bellow } 10 \mathrm{~m}\end{array}$ & $\begin{array}{l}\text { Lissotriton vulgaris, Bombina bombina, Pelobates fuscus, } \\
\text { Bufotes viridis, Natrix natrix }\end{array}$ \\
\hline Mărculești & $\begin{array}{l}\text { N44 } 33.764 \\
\text { E27 } 32.406\end{array}$ & Bombina bombina, Bufotes viridis \\
\hline Ghimbășani & $\begin{array}{l}\text { N44 } 33.733 \\
\text { E27 } 29.028\end{array}$ & Dolichophis caspius \\
\hline Alexeni & $\begin{array}{l}\text { N } 4440.564 \\
\text { E } 2643.326\end{array}$ & Natrix natrix \\
\hline Bărbătescu & $\begin{array}{l}\text { N } 4436.180 \\
\text { E } 2648.163\end{array}$ & Pelophylax ridibundus, Lacerta viridis, Lacerta agilis \\
\hline Făcăeni & $\begin{array}{l}\text { N } 4434.550 \\
\text { E } 2753.740 \text { bellow } 10 \mathrm{~m}\end{array}$ & Bufotes viridis \\
\hline
\end{tabular}




\section{DISCUSSION}

We have found 12 amphibian species (Triturus cristatus, Triturus dobrogicus, Lissotriton vulgaris, Bombina bombina, Bombina variegata, Pelobates syriacus, Pelobates fuscus, Bufotes viridis, Hyla orientalis, Rana dalmatina, Pelophylax ridibundus, Pelophylax lessonae), two amphibian hybrids (Triturus cristatus x $T$. dobrogicus and Pelophylax kl. esculentus) and five reptile species (Emys orbicularis, Lacerta agilis, Lacerta viridis, Natrix natrix, Dolichophis caspius) distributed across the Ialomița County; most of the records tabulated above are new. The only species firstly recorded for this county are Triturus cristatus and Rana dalmatina. A correspondence between the recent scientific names used for these species, according to currently prevailing taxonomic opinion, and the "older" scientific names used in Romanian environment legislation, is given in table 2 below, to avoid possible confusion regarding protective status.

The most common amphibians are Pelophylax ridibundus (66 locations) and Bombina bombina (58 locations, which is rejoicing as the species is protected under the European Habitats Directive Annex II); Bufotes viridis is also common with 46 records. The most common reptiles are Lacerta viridis (26 locations) and Lacerta agilis (18 locations). Emys orbicularis, also a protected species under the European Habitats Directive Annex II, was found in 10 locations, which is remarkable.

We did not record Bufo bufo, Podarcis tauricus (these were, however, found by us in the floodplain of Balta Ialomiței - Iftime \& Iftime, 2007) or Natrix tessellata. The

Correspondence between current and older names in use for observed taxa.

\begin{tabular}{|l|l|l|}
\hline $\begin{array}{l}\text { Currently used scientific name } \\
\text { (Speybroeck et al., 2016) }\end{array}$ & $\begin{array}{l}\text { Scientific name appearing in Romanian } \\
\text { environment legislation (L49/2011) }\end{array}$ & Observations \\
\hline Triturus cristatus & $\begin{array}{l}\text { Triturus cristatus (Triturus } \\
\text { cristatus cristatus) }\end{array}$ & \\
\hline Triturus dobrogicus & $\begin{array}{l}\text { Triturus dobrogicus (Triturus } \\
\text { cristatus dobrogicus) }\end{array}$ & \\
\hline Bombina bombina & Bombina bombina & \\
\hline Bombina variegata & Bombina variegata & \\
\hline Pelobates fuscus & Pelobates fuscus & \\
\hline Pelobates syriacus & Pelobates syriacus & \\
\hline Bufotes viridis & Bufo viridis & \\
\hline Hyla orientalis & Hyla arborea & \\
\hline Rana dalmatina & Rana dalmatina & A “klepton" \\
\hline Pelophylax ridibundus & Rana ridibunda & \\
\hline Pelophylax lessonae & Rana lessonae & \\
\hline Pelophylax $\mathrm{kl}$ esculentus & Rana esculenta \\
\hline Emys orbicularis & Emys orbicularis & \\
\hline Lacerta agilis & Lacerta agilis & \\
\hline Lacerta viridis & Lacerta viridis & \\
\hline Natrix natrix & - & Coluber caspius \\
\hline Dolichophis caspius & & \\
\hline
\end{tabular}



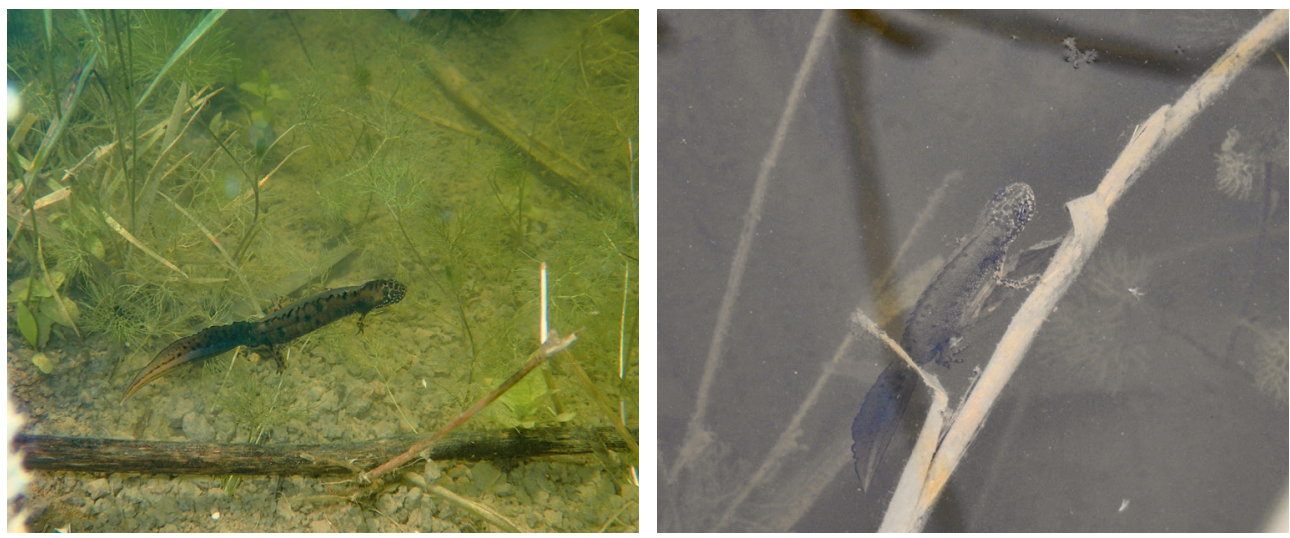

Fig. 1 - A: Triturus dobrogicus, adult male, Bordușani, 2016; B: morphologically Triturus cristatuslike adult male from hybrid population, Armășești (photos by A. Iftime)
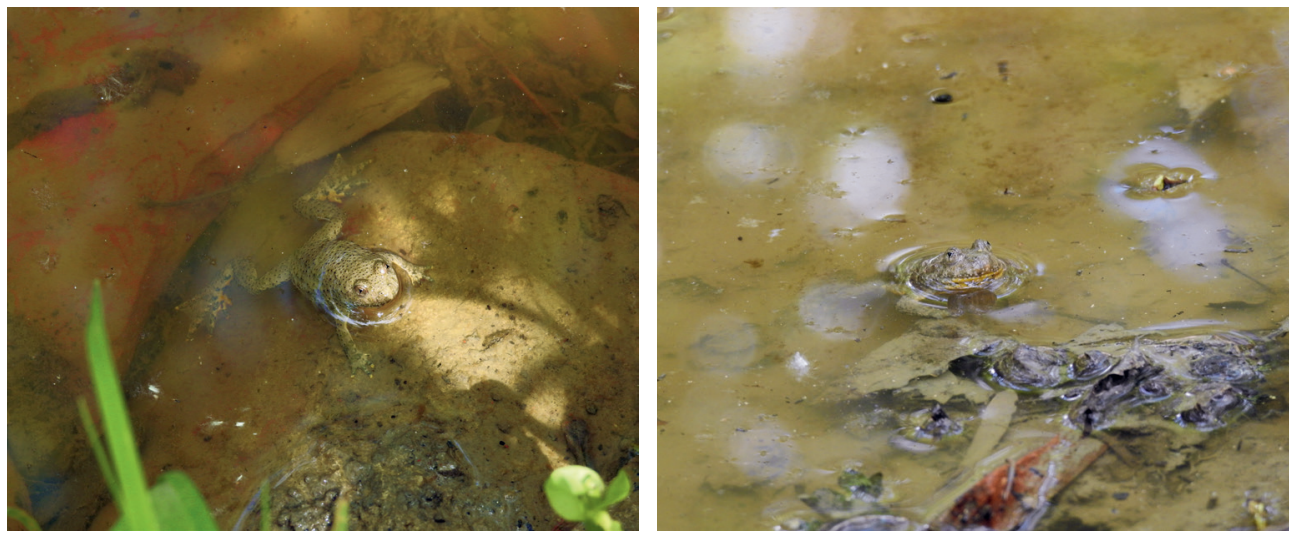

Fig. 2 (A \& B) - Bombina variegata, adult female, near Rădulești (Brazii), 2016 (photos by A. Iftime);

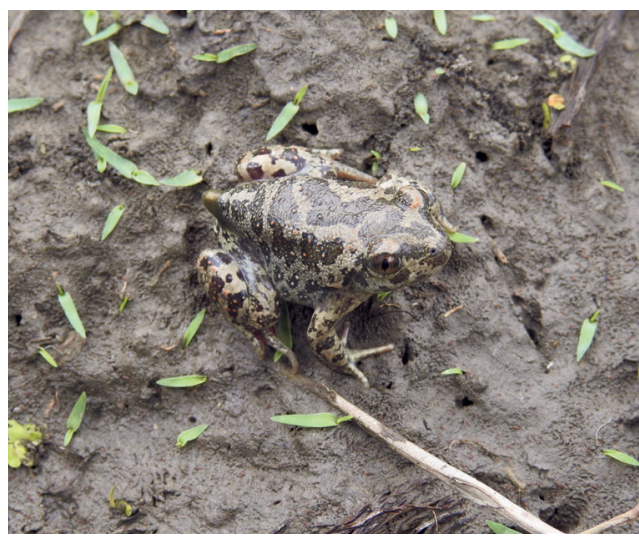

Fig. 3 - Pelobates syriacus, metamorph, Munteni-Buzău, 2016 (photo by A. Iftime). 

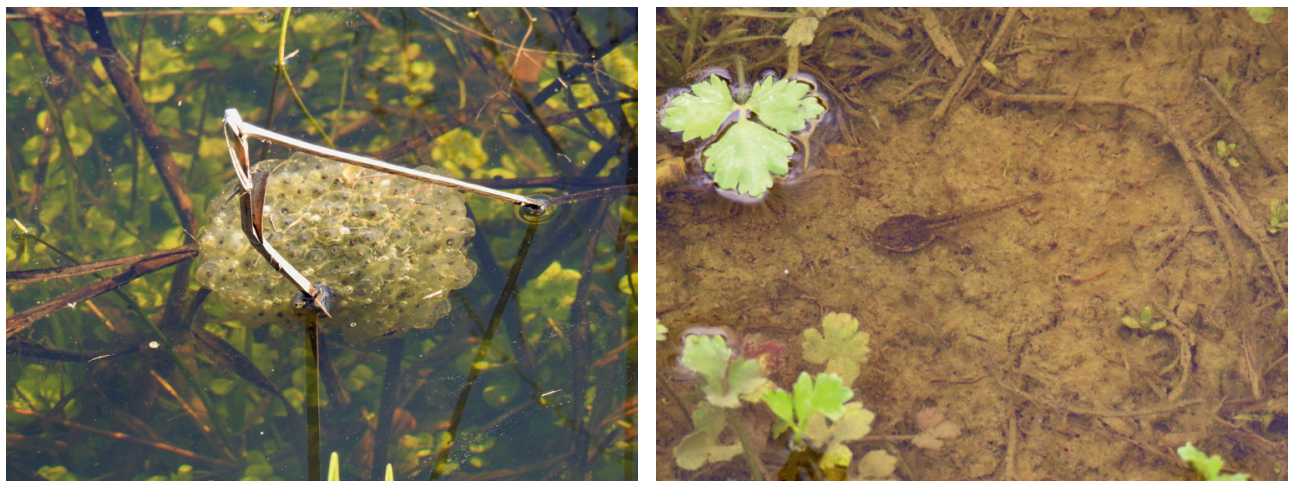

Fig. 4 -Rana dalmatina: A, egg mass, Orezu, 2016; B, larva, Coșereni, 2016 (photos by A. Iftime);

lack of records for $N$. tessellata is in agreement to what we found in the neighbouring Teleorman county (Iftime \& Iftime, 2016). However, Lacerta agilis appears to be as frequent in Ialomița as we found it to be scarce in other southern regions of Romania: Giurgiu (Iftime \& Iftime, 2008) and Teleorman (Iftime \& Iftime, 2016).

In the case of Crested Newts (the Triturus cristatus species group) we have found 13 new localities for morphologically pure $T$. dobrogicus, significantly extending the range of this species in the Romanian Plain (compare to Covaciu-Marcov et al., 2009; Cogălniceanu et al., 2013a; Wielstra et al., 2016). Some specimens have an atypical morphology, yet within the variation of T. dobrogicus (Wielstra et al., 2016) and not suggesting hybridization. In one locality the newts were found to be morphologically intermediate between $T$. dobrogicus and T. cristatus and might be presumed to be of hybrid origin; close to this we also found a population of (apparently) morphologically pure T. cristatus.

For Bombina variegata - a typically hill- and mountain-dwelling species we found one new record, which is technically outside Ialomița County, although in an area which on most maps is shown within it; anyway it is extremely close to the county border, and should be mentioned for the zoogeographical interest, as it
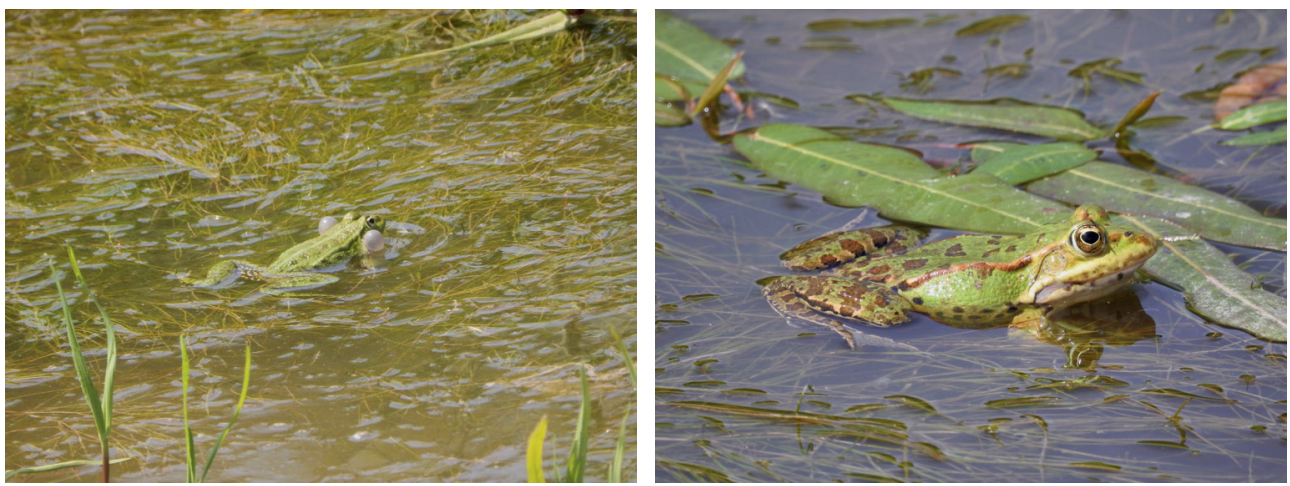

Fig. 5 - A: Pelophylax kl. esculentus, adult male, Rădulești (Brazii), 2016 (photo by O. Iftime); note the white vocal sacs, yellow coxal spots, relatively large metatarsal tubercle; B: Pelophylax lessonae, adult male, Rădulești (Brazii), 2016 (photo by A. Iftime); note the large metatarsal tubercle. 


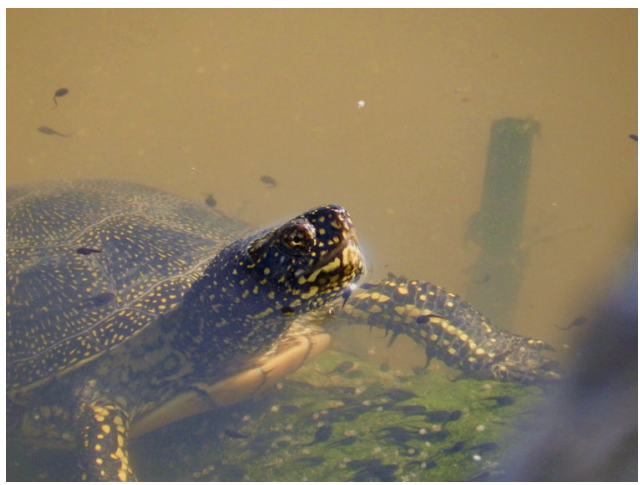

Fig. 6 -Emys orbicularis, adult, with Bufo viridis larvae, Gheorghe Doja, 2016 (photo by O. Iftime).

is one of the very few records of this species in the Romanian Plain (compare to Cogălniceanu et al., 2013a).

Also of interest are the five new locations for Pelobates syriacus, considerably extending the distribution towards the south and west in the Ialomița valley area (compare to Cogălniceanu et al., 2013a; Székely et al., 2013; Török, 2014). Some places (e. g. Munteni-Buzău) offered the opportunity to observe, during several visits, the development of the tadpoles of this species up to metamorphosis. Many authors (e.g. Fuhn, 1960; Sidorovska et al., 2002; Székely et al., 2013) claim that P. syriacus tadpoles cannot be accurately distinguished from P. fuscus tadpoles; however, see Speybroeck et al., 2016 - whose data our observations tend to confirm.

Rana dalmatina was firstly recorded for this county. While not unexpected in the forests in the west of the county, its occurrence along the Ialomiţa River is interesting.

In the Pelophylax genus (the "West Palearctic Water Frogs" or "Green Frogs"), we found all three forms that are present in Romania - the two species $P$. ridibundus and $P$. lessonae, and their hybridogenetic kleptotaxon $P$. kl. esculentus. We note that $P$. ridibundus is the most widely distributed and abundant; of our 65 records of Pelophylax frogs, 42 were of $P$. ridibundus alone, 15 of $P$. ridibundus and $P$. kl. esculentus, three of all three species together, and two where we only noticed $P$. ridibundus and $P$.

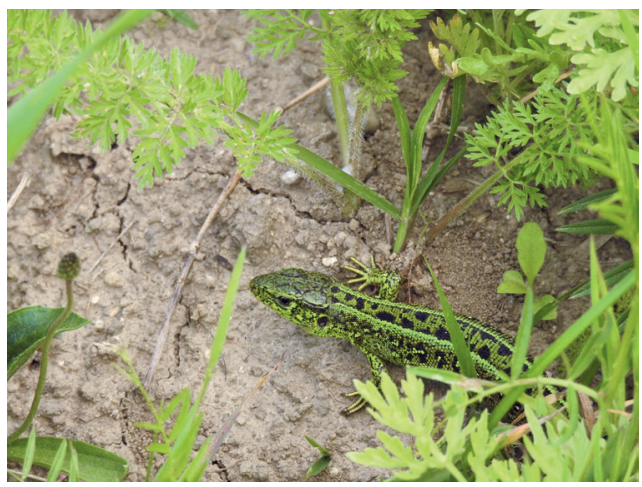

Fig. 7 - Lacerta agilis, adult male, Bărbulești, 2016 (photo by O. Iftime). 


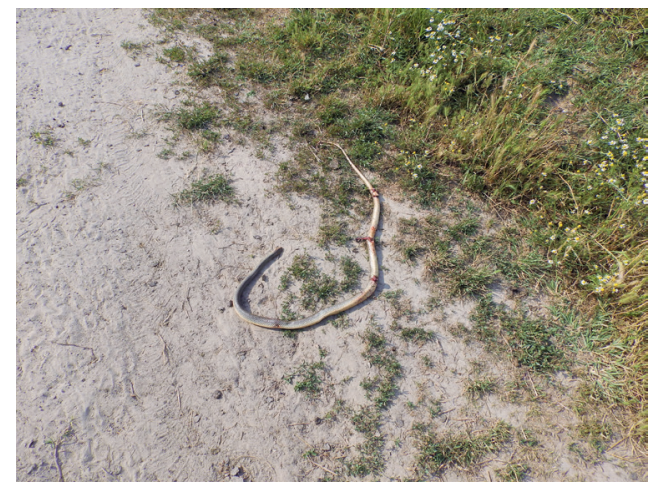

Fig. 8 - Dolichophis caspius, killed adult, Piersica, 2016 (photo by O. Iftime).

lessonae (of course, we do not exclude the possibility of the hybrid being also present there). More research would be needed to assess the presence and chart the distribution of R-E and R-L-E (and possibly other) hybridogenetic systems in this area. At least in one place (Rădulești/Brazii, observed in late April 2016) coexisting P. ridibundus, $P$. lessonae and $P$. kl. esculentus appeared to be involved together in reproductive activities, sound displays and associated fights, possibly indicating a R-L-E system.

Among reptiles, the Caspian Whip Snake (Dolichophis caspius) was found in a number of nine points, significantly extending its known range in the Romanian plain (compare to Fuhn \& Vancea, 1961; Iftime \& Iftime, 2007; Cogălniceanu et al., 2013b; Sahlean et al., 2014) and thus confirming the presence of this species in the central Ialomita plain, where it was indicated by the old record of Fuhn \& Vancea (1961) from Ghimpați (this record was, however, not included in recent synthetic works, e.g. Cogălniceanu et al., 2013b).

We also note that, despite intense anthropogenic modification (mainly expressed in large agricultural monocultures and the associated drainage and irrigation works) the herpetofauna has managed to survive in considerable numbers, both of species and individuals. Many amphibians use the ditches and canals made for drainage (in the wetlands) and for irrigation (in agricultural areas). Although irrigation ditches and canals are not in agricultural use anymore, they still drain and collect enough water to ensure successful reproduction for numerous amphibians, including protected species (e.g. Triturus dobrogicus, T. cristatus, Bombina bombina).

Another interesting observation pertaining to amphibian ecology is that apparently no amphibians live in some parts of the highly mineralized Amara Lake.

Another important anthropogenic impact is the depositation of garbage, which is largely haphazard and too often takes the form of dumping into waterbodies whatever must be thrown out. As a result, some waterbodies, including some used by a large number of amphibians (e.g. at Ciochina), are studded or almost choked up with household garbage. On the brighter side, contamination of natural habitats (including waterbodies) with fertilizer and pesticide runoff from agriculture appears to be low (inasmuch as we could observe) - probably reflecting a more careful use of these substances that at sometimes in the past, albeit this is likely driven more by their cost than by concern for the environment.

On the whole, despite such impacts, the Ialomiţa County offers good conditions for the long-term persistence of amphibian and reptile species, including such species 
which are protected under the European Habitats Directive Annex II as Triturus dobrogicus, T. cristatus, Bombina bombina and Emys orbicularis, providing good opportunities for conservation.

\section{REFERENCES}

COGĂLNICEANU, D. (1997) Practicum de ecologie a amfibienilor: Metode şi tehnici în studiul ecologiei amfibienilor. Ed. Universității din București, 122 pp. (in Romanian)

COGĂLNICEANU, D., P. SZÉKELY, C. SAMOILĂ, R. IOSIF, M. TUDOR, R. PLĂIAȘU, F. STĂNESCU, L. ROZYLOWICZ (2013a) Diversity and distribution of amphibians in Romania. ZooKeys, 296: 35-57.

COGĂLNICEANU, D., L. ROZYLOWICZ, P. SZÉKELY, C. SAMOILĂ, F. STĂNESCU, M. TUDOR, D. SZÉKELY, R. IOSIF (2013b) Diversity and distribution of reptiles in Romania. ZooKeys, 341: 49-76.

COVACIU-MARCOV, S.-D., A.-Ș. CICORT-LUCACIU, I. SAS, A. FILIMON (2009) Notes on the herpetofauna of south-eastern plain areas from Romania. Universitatea din Craiova, seria Biologie, Horticultură, Tehnologia prelucrării produselor agricole, Ingineria mediului, 14 (XLX): 451-456.

FUHN, I. (1960) Amphibia. In: Fauna R.P.R., 14, 1, Edit. Academiei R.S.R., Bucureşti. (in Romanian)

FUHN, I., ŞT. VANCEA (1961) Reptilia. In: Fauna R.P.R., 14, 2, Edit. Academiei Române. Bucureşti. (in Romanian)

IFTIME, A., O. IFTIME (2007) Some records of the herpetofauna of the Danube floodplains in the Balta Ialomiței area (Romania). Travaux du Muséum National d’Histoire Naturelle "Grigore Antipa", 50: 273-281

IFTIME, A., O. IFTIME (2008) Observations on the herpetofauna of the Giurgiu county. Travaux du Muséum National d'Histoire Naturelle "Grigore Antipa", 51: 209-218.

IFTIME, A., O. IFTIME (2016) Contributions to the knowledge on the amphibians and reptiles of Teleorman county (southern Romania). Travaux du Muséum National d'Histoire Naturelle "Grigore Antipa", 58(1-2): 63-71.

MÂCIU, M., CHIOREANU, A., VĂCARU, V. (ed.), 1982. Enciclopedia geografică a României. Ed. Ştiinţifică şi Enciclopedică, Bucureşti, 847 pp. (In Romanian)

SAHLEAN, T.C., I. GHERGHEL, M. PAPEȘ, A. STRUGARIU, Ș.R. ZAMFIRESCU (2014) Refining climate change projections for organisms with low dispersal abilities: a case study of the Caspian Whip Snake. PLoS ONE 9(3): e91994. doi:10.1371/journal.pone.0091994.

SIDOROVSKA, V., K. LJUBISAVLJEVIC, G. DŽUKIĆ, M.L. KALEZIĆ (2002) Tadpole morphology of two spadefoot toads (Pelobates fuscus and P. syriacus) (Amphibia, Anura, Pelobatidae). Spixiana, 25: 183-191.

SOS, T. (2011) În obiectiv: Țestoasa de apă europeană, Emys orbicularis. Asociaţia Ecouri Verzi, Cluj-Napoca (in Romanian).

SPEYBROECK, J., W. BEUKEMA, B. BOK, J. VAN DER VOORT, I. VELIKOV (2016) Field Guide to the Amphibians and Reptiles of Britain and Europe (British Wildlife Field Guides). Bloomsbury Natural History, London/New York.

SZÉKELY, P., R. IOSIF, D. SZÉKELY, F. STÄNESCU, D. COGĂLNICEANU (2013) Range extension for the Eastern spadefoot toad Pelobates syriacus (Boettger, 1889) (Anura: Pelobatidae). Herpetology Notes 6: 481-484.

TÖRÖK , ZS., (2001) Herpetological observations in the lower Danube area (Calafat-Călăraşi sector). Studii şi cercetări, Biologie, Universitatea din Bacău, 6, 115-119.

TÖRÖK, Z.S. (2014) New data on the distribution of Pelobates syriacus in Romania and first record of the species in Vrancea county. BSB Net-Eco International Conference, Environmental aspects and available scientific tools for Black Sea Basin protection "Deltas and wetlands"Tulcea, 15-17 September 2014.

WIELSTRA, B.,J. VÖRÖS, J.W. ARNTZEN (2016) Is the Danube crested newt Triturus dobrogicus polytypic? A review and new nuclear DNA data. Amphibia-Reptilia, 37 (2): 167-177.

*** L 49/2011. Lege (nr. 49/2011) pentru aprobarea Ordonanţei de urgenţă a Guvernului nr. 57/2007 privind regimul ariilor naturale protejate, conservarea habitatelor naturale, a florei şi faunei sălbatice. Monitorul Oficial al României, 262: 2-12 (in Romanian).

*** Council Directive 92/43/EEC of 21 May 1992 on the conservation of natural habitats and of wild fauna and flora. $66 \mathrm{pp}$. 